\title{
Morphing UAV Pareto Curve Shift for Enhanced Performance
}

\author{
Michael T. Rusnell * $\quad$ Shawn E. Gano * $\quad$ Victor M. Pérez ${ }^{\dagger}$ \\ John E. Renaud $\ddagger$ Stephen M. Batill $\ddagger$ \\ Department of Aerospace and Mechanical Engineering \\ University of Notre Dame \\ Notre Dame, Indiana \\ Email: John.E.Renaud.2@nd.edu
}

\begin{abstract}
Research in unmanned aerial vehicles (UAVs) has grown in interest over the past couple decades. Historically, UAVs were designed to maximize endurance and range, but demands for UAV designs have changed in recent years. In addition to the traditional demands for endurance and range, today customer demands include maneuverability. Therefore, UAVs are being designed to morph, to change their geometrical shape during flight, for enhanced maneuvering capability. In this investigation the morphing UAV concept under study is referred to as the buckle wing. The design of the buckle-wing airfoil geometries is posed as a multilevel, multiobjective optimization problem. This buckle-wing design problem includes two competing objectives of maneuverability and long range/endurance. Multiobjective problems have many optimal solutions each depicting a different compromise scenario. Each optimal solution is a Pareto point, and the set of all these points represents the Pareto curve. This is a powerful means of showing the global picture of the solution field. The goal of this paper is to explore and compare the Pareto curves of the buckle-wing UAV to that of a conventional non-morphing UAV. In order to make this performance comparison, Compromise Programming is used as the optimizing method, and the VortexPanel Method is used in calculating the aerodynamics. The buckle-wing UAV's enhanced capabilities are demonstrated both quantitatively and graphically.
\end{abstract}

\section{Nomenclature}

$\begin{array}{ll}\alpha & \text { Angle of attack } \\ c d & \text { Drag coefficient } \\ c l & \text { Lift coefficient } \\ M a & \text { Mach Number } \\ \text { ReL } & \text { Reynolds Number }\end{array}$

*Graduate Research Assistant, Student Member AIAA.

$\dagger$ Research Associate, Member AIAA.

${ }^{\ddagger}$ Professor, Associate Fellow AIAA. 


\section{Introduction}

\section{A. Morphing UAV Background}

Research in unmanned aerial vehicles (UAVs) has grown over the past couple of decades. Interest in UAVs is growing in interest because of the dangerous missions they can accomplish without the risk of human life. In particular, UAVs are of great interest to the U.S. Air Force, and funding from the Air Force makes the research of this project possible. UAV missions include video and IR surveillance, relay communication links, and detection of biological, chemical, or nuclear materials. These missions are autonomously controlled or piloted by remote control.

Demands for UAVs have changed in recent years. Historically, UAVs were designed to maximize endurance and range. In addition to customer needs for endurance and range, today's customer also demands maneuverability. This is especially important if the UAV encounters any resistance during the mission. A typical mission for the newer multi-role UAV includes takeoff, cruising to the desired location as efficiently as possible, maneuvering to escape problematic encounters, cruising back, and landing. During takeoff, evasive maneuvers, and landing, high lift is required and less emphasis is put on vehicle drag. For cruising however, maximizing range and endurance are desired making the lift-to-drag ratio important. The capability of obtaining maneuverability and obtaining endurance and range in the same design presents a problem because of the inherent tradeoff between the two.

Whereas the conventional UAV maximizes endurance and range, the UAV considered in this investigation is being designed to morph for an enhanced capability for maneuverability. The UAV is designed to morph; that is, it is designed to change geometrical shape during flight. This project investigates the unique bucklewing UAV design project in the Aerospace and Mechanical Engineering Department at Notre Dame. ${ }^{2}$ Figure 1 depicts a simulated buckle wing in flight.

Programs for morphing aircraft research have been emerging in recent years. DARPA announced its Morphing Aircraft program in Aviation Week and Space Technology in April 2002. ${ }^{11}$ NASA also has a large Aircraft Morphing program. ${ }^{13}$ A team from Purdue University, supported by NASA, recently generated 35 mission concepts for morphing aircraft. ${ }^{5}$ Furthermore, the Purdue team presented a remarkable concept looking at morphing as an independent variable. ${ }^{6}$ The team's approach uses aircraft performance, size, and weight as functions of morphing to size an aircraft. Another interesting area of morphing aircraft research examines the flying mechanics of birds and apply them as morphing principles on larger air vehicles. ${ }^{1}$ On the system level, they examine the effects of variable lift-to-drag ratio and specific fuel consumption of the vehicle in cruising flight.

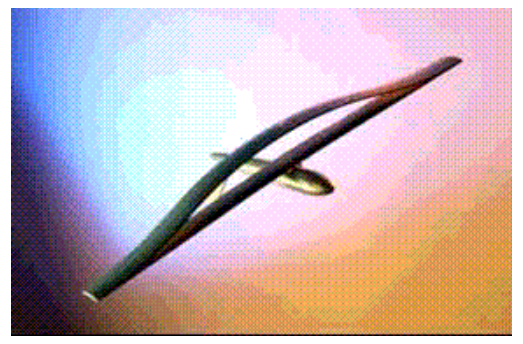

Figure 1. Buckle-Wing UAV

The morphing concept of the buckle-wing UAV has the ability to change its wing configuration from a single wing into two wings joined at each end. The research focus of for this project consists of designing the buckle-wing UAV's airfoil cross-sections. The probrem is posed as a single airfoil which splits into two airfoils when morphed. The first configuration is called fused. It consists of a single airfoil, and its objective is to optimize endurance and range via lift-to-drag $\mathrm{cl} / \mathrm{cd}$. The second configuration is called split, or the buckle wing, because it consists of that same single fused airfoil now only split into two airfoils. This configuration 
is designed to optimize maneuverability via lift $c l$.

The buckle wing has many advantages over a conventional UAV design, in that the tradeoff between maneuvering, and endurance and range can be somewhat decoupled. The buckle-wing concept allows greater performance of each capability as compared to any single design with competing objectives. However, it also presents new design challenges. These two competing objectives create a multiobjective problem for the two wing configurations.

The design problem is formulated as a multiobjective optimization problem consisting of two competing objectives. Maximizing one objective occurs at the expense of the other, or increasing one objective results in a decrease in the other. In such cases the designers must make a compromise in order to obtain satisfactory objective values. A multiobjective optimization problem has many optimal solutions each depicting a different compromise scenario. Each solution presents a point, and the set of all these points represents the Pareto curve. The Pareto curve is a powerful graphical tool to show the global tradeoff picture. There are a number of procedures proposed in research journals and textbooks for generating a compromise point. The Pareto curves of the buckle-wing UAV and a conventional UAV are generated from the method of Compromise Programming ${ }^{9}$ in this investigation.

The goal of this paper is to explore and compare the Pareto curve of the buckle-wing UAV with that of the conventional UAV in order to quantitatively demonstrate the buckle wing's enhanced capabilities. The superiority of the buckle wing will be immediately identifiable.

\section{B. Compromise Programming Background}

Goal Programming (GP) is the background for the method used in this research. Based on the works of Steuer, ${ }^{8}$ the variation of GP used in this investigation is described in Tappeta ${ }^{9}$ and otherwise known as Compromise Programming.

Compromise Programming (CP) is a powerful method of solving multiobjective optimization problems. $\mathrm{CP}$ has the advantage of generating efficient solutions even when the Pareto curve is nonconvex. It is based on minimization of the distance between a feasible point and an unattainable point called the ideal point. The feasible point $\bar{f}_{i}$ is the feasible optima the designer wishes to obtain. It is actually a vector quantity and also known as the aspiration point. The unattainable, ideal point $f_{i}^{u}$ is also called the utopian point. This too is a vector. As its name suggests it contains the unattainable, ideal solution of each objective function.

The CP optimization problem is described as

$$
\begin{array}{ll}
\text { Minimize } & z+\alpha \sum_{i=1}^{m} w_{i} f_{i}(x) \\
\text { Subject to }: & z \geq w_{i}\left(f_{i}(x)-f_{i}^{u}\right), i=1, \ldots, m \\
& g_{j}(x) \geq 0, j=1, \ldots, p \\
& h_{k}(x)=0, k=1, \ldots, q \\
& x_{l}^{u b} \geq x_{l} \geq x_{l}^{l b}, l=1, \ldots, n .
\end{array}
$$

In this standard form of the CP problem, the objective functions $F(x)=\left(f_{1}(x), f_{2}(x), \ldots, f_{m}(x)\right)$ must be minimized. The weights $w_{i}$ are determined by $w_{i}=1 /\left(\bar{f}_{i}-f_{i}^{u}\right)$, and are determined before solving the CP problem. $\alpha$ is an optimization parameter set to a sufficiently small positive number such as $10^{-6}$.

The solution of the CP problem produces a single point. This solution is a Pareto point if it meets the Pareto optimality condition. A vector of $x^{*}$ is Pareto optimal if there exists no feasible vector $\mathrm{x}$ which would decrease some objective function without causing a simultaneous increase in at least one objective function.

3 OF 15 


\section{Research}

\section{A. Compromise Programming Implementation}

Because the UAV airfoil design problem seeks to maximize the objective functions, the CP problem formulation for minimization must be changed. Several changes must take place to change the formulation into a maximizing one. First, maximizing an objective function is analogous to minimizing the objective function's negative value. Mathematically, this is stated as

$$
\text { Maximize } F(x)=\text { Minimize }-F(x) \text {. }
$$

Additionally, the utopian point $f_{i}^{u}$ is no longer the ideal minimal point, but it is a maximum ideal point greater than any value the designer could hope to achieve. Therefore, the weights have to be changed as well. The weights become $w_{i}=1 /\left(f_{i}^{u}-\bar{f}_{i}\right)$.

$F(x)=f_{1}(x), f_{2}(x) . f_{1}$ represents the first objective for endurance/range $c l / c d$, and $f_{2}$ represents the second objective for maneuverability $\mathrm{cl}$. Since this is a maximizing problem, the $\mathrm{CP}$ formulation is

$$
\begin{array}{ll}
\text { Minimize } & z-\alpha \sum_{i=1}^{m} w_{i} f_{i}(x) \\
\text { Subject to }: & z \geq w_{i}\left(f_{i}^{u}(x)-f_{i}\right), i=1, \ldots, m \\
& g_{j}(x) \geq 0, j=1, \ldots, p \\
& h_{k}(x)=0, k=1, \ldots, q \\
& x_{l}^{u b} \geq x_{l} \geq x_{l}^{l b}, l=1, \ldots, n
\end{array}
$$

Choosing a specific set of weights defines the optimization problem. The problem then can be solved for a solution according to those weights or aspiration values. If an optimal solution is found, and its weights were chosen properly, the solution represents a Pareto point. All these points, corresponding to the different weights used in obtaining the optimal solutions, depict the Pareto curve.

Aspiration values should be chosen that are obtainable. The idea is to choose values which are just beyond the previous solution in order to find the true Pareto point. Choosing aspiration values which are too low will generate a solution, but generally this will not be a Pareto point. The aspiration targets are effectively used as weights in CP multiobjective optimization. Aspiration values were chosen such that the Pareto curve could be generated in the shortest number of trials.

In the conventional UAV design problem generally $c l$ values around 2.0 could be achieved depending upon the airfoils selected as basis functions (cf. section D). For the buckle-wing problem achievable $c l$ values are closer to 3.0. In both cases $c l / c d$ values could achieve values of around 150 .

The process is very intuitive and important. In order to achieve high values for one of the objectives, the corresponding aspiration values would need to be set high at the expensive of the other aspiration value. However, if the other aspiration value is too low, then the problem may converge without generating a Pareto point. Because computational time is the limiting variable in performing this research, it is really important to set the aspiration values wisely in order to minimize the number of times necessary to run the code to generate the Pareto curve.

\section{B. Conventional UAV Airfoil Design Study}

The conventional UAV airfoil design study is a single-level, multiobjective problem implemented in two variations. The first variation makes use of eight design variables representing the weights of the eight basis functions, and the second variation makes use of only three basis functions. Each also makes use of the pseudo design variable $z$. Each optimization problem also loads the data for the spline of each basis function. In the case consisting of eight design variables, the objective is to determine the three most dominant basis functions. Then, the second variation is run for the comparison study of the conventional and buckle-wing Pareto curves. Both variations minimize the objective function

4 OF 15

American Institute of Aeronautics and Astronautics 


$$
F=z-\alpha\left(w_{1} f_{1}+w_{2} f_{2}\right)
$$

where $z$ is the pseudo design variable, $f_{1}=c l / c d$, and $f_{2}=c l$. Unlike the buckle-wing study, the conventional study involves only a single wing. Hence, $c l / c d$ and $c l$ are the values for this single wing. The lower bounds of the design variables are 0 . The weights of the basis functions are not allowed to be negative to ensure a valid design. The upper bounds of the design variables are 1.5. This number is chosen to ensure that the airfoils do not become too thick. These airfoils are appropriate selections for aircrafts such as the UAV, and allowing them to become much thicker would not be suitable for design. Likewise, another constraint guarantees that the sum of the design variables does not exceed 1.5 for the same reason. The other constraints are the two $\mathrm{CP}$ constraints. These constraints tend to drive the optimization process through the design variable $z$, which is minimized as part of the objective. The optimizer calls the aerodynamic analysis code (i.e., the panel method).

The aerodynamic analysis code calculates lift and drag. It is a modified version of Pablo, ${ }^{12}$ which was developed in 1999 in Stockholm, Sweden. The aerodynamic analysis code uses a two-dimensional panel method. It consists of a two-step process. First, lift is calculated. Then, using this result an estimate for drag is found. This process takes approximately one-half second for a single analysis. A two-dimensional vortex panel method ${ }^{3}$ calculates drag sectional lift and the pressure distribution. A boundary-layer model calculates the drag employing Thwaites' equations for the laminar region and Head's equations for turbulent region. ${ }^{4}$ The drag coefficient is then computed using the Squire-Young formula. ${ }^{7}$

\section{Buckle-Wing Problem}

The buckle-wing problem is a multilevel, multiobjective optimization problem. The problem consists of three design variables and a fourth pseudo design variable. The upper-level objective function is to minimize

$$
F=z-\alpha\left(w_{1} f_{1}+w_{2} f_{2}\right),
$$

where $z$ is the pseudo design variable, $f_{1}$ is $c l / c d_{f u s e d}$ or $c l / c d$ in the fused configuration, and $f_{2}$ is $c l_{\text {split }}$ or the $c l$ in the buckle-wing (i.e., split) configuration. The same constraints from the conventional problem are also imposed upon this problem. The upper-level optimizer calls upon the aerodynamic analysis code to calculate the $\mathrm{cl} / \mathrm{c} d_{f u s e d}$, and $c l_{f u s e d}$, and the optimizer calls upon the lower-level optimizer to optimize a cut and calculate the $c l / c d_{\text {split }}$ and $c l_{\text {split }}$.

The lower-level optimizer consists of three design variables representing the control points. The control points lie upon a cubic spline curve used to cut the fused airfoil. This suboptimization problem additionally contains three fixed points, which also lie upon the cubic spline curve. They are located at the leading edge, the trailing edge, and one near the trailing edge to ensure that the split airfoil has a sharp trailing edge.

The suboptimizer produces a cut based on the objective and constraints. The objective is to maximize the buckle wing's split configuration $\mathrm{cl}$. The lower bounds are set to ensure that the lower split airfoil contains at least $30 \%$ of the original airfoil. The upper bounds ensure that the upper split airfoil also contains at least $30 \%$ of the original airfoil. Another constraint is added to guarantee that the buckle wing's $\mathrm{cl} / \mathrm{cd}$ is greater than 60 . The suboptimizer calls upon a subroutine to make the cut and calls upon the aerodynamic analysis code to calculate the buckle wing's $c l$ and $c l / c d$. This suboptimization process was developed in Gano. $^{2}$

This optimization problem has many parameters. It is important to keep in mind that the goal of this project is to compare the Pareto curves of the buckle-wing UAV to that of a conventional UAV. Therefore, varying parameters is superfluous to achieving the main goal. Consequently, the Reynolds number ReL, angle of attack $\alpha$, and Mach numbers $M a$ were kept constant and given real values which the UAV would see in its mission. These values are shown in Table 1. These values correspond to realistic ones experienced in UAV applications. 
Table 1. Optimization Parameters and Their Values

\begin{tabular}{|c|c|}
\hline Parameter & Value \\
\hline$R e L$ & $5 \mathrm{e} 5$ \\
\hline$\alpha$ & 2 \\
\hline$M a$ & 0.3 \\
\hline
\end{tabular}

\section{Basis Functions}

Basis functions are used to describe the shape of the airfoil. This is a technique given by Vanderplaats. ${ }^{10}$ An airfoil is typically represented by a cubic spline and defined by over 100 data points. Because these points are so numerous, making each of these points a design variable is impractical for computational fluid dynamics (CFD) or other computational analyses such as panel codes. Therefore, basis functions are used, where each basis function represents a preexisting airfoil. Each one is given a weight, and each weight represents a design variable.

Cubic splines are necessary to convert the preexisting airfoil shape into a basis function used in the optimization problem. The University of Illinois at Urbana-Champaign has an excellent link to an airfoil database site. This site is http://www.aae.uiuc.edu/m-selig/ads/cood_database.html. The airfoils on this website are described by a set of data points. Each different type of airfoil varies in the number of data points given and in airfoil orientation. For this research project cubic splines are used to convert the unstandardized, varying data into a 141 data points with the same orientation. Thus, each airfoil taken from the website is transformed into a basis function sharing the same number of points and values in $x$ coordinates.

\section{Results}

\section{A. Conventional UAV Study}

A study of the conventional UAV is absolutely necessary. It provides a basis of comparison for the bucklewing UAV design. In order to make a true comparison, the same criteria have to be implemented in both studies. Then, an evaluation can be made quantitatively and graphically to demonstrate the effectiveness of the buckle-wing UAV design.

The conventional UAV problem is, quite understandably, less complicated than the buckle-wing problem. The optimization problem for the conventional UAV involves a multiobjective design of a single airfoil. The conventional UAV problem optimizes both range/endurance and maneuverability for the single wing. In contrast, the buckle wing involves multilevel, multiobjective optimization. It optimizes the fused airfoil for its range and endurance performance and the split airfoil for maneuverability. Consequently, the conventional UAV optimization problem is computationally much less expensive and generating its Pareto curve takes significantly less time. Hence, the conventional UAV problem was studied first.

The conventional UAV problem contains eight airfoil basis functions. Because the conventional wing problem is not computationally expensive compared to the buckle wing, it can include more design variables. Eight sundry airfoils were chosen for the first single-wing optimization problem shown in Figure 2. Basically, these eight were chosen because they cover a gambit of families of airfoil shapes. The only criterion for selecting them was that they are designed for small aircraft use. This meant that the airfoils had to be designed for low Reynolds number, and that they had to be thin airfoils.

Because the buckle-wing problem is computationally more expensive, only three of the eight airfoils could be used for a Pareto comparison with the buckle-wing problem. Performing the conventional study first also helped in order to discover which three airfoils should be used as basis functions for the buckle wing. Basis

6 of 15

American Institute of Aeronautics and Astronautics 

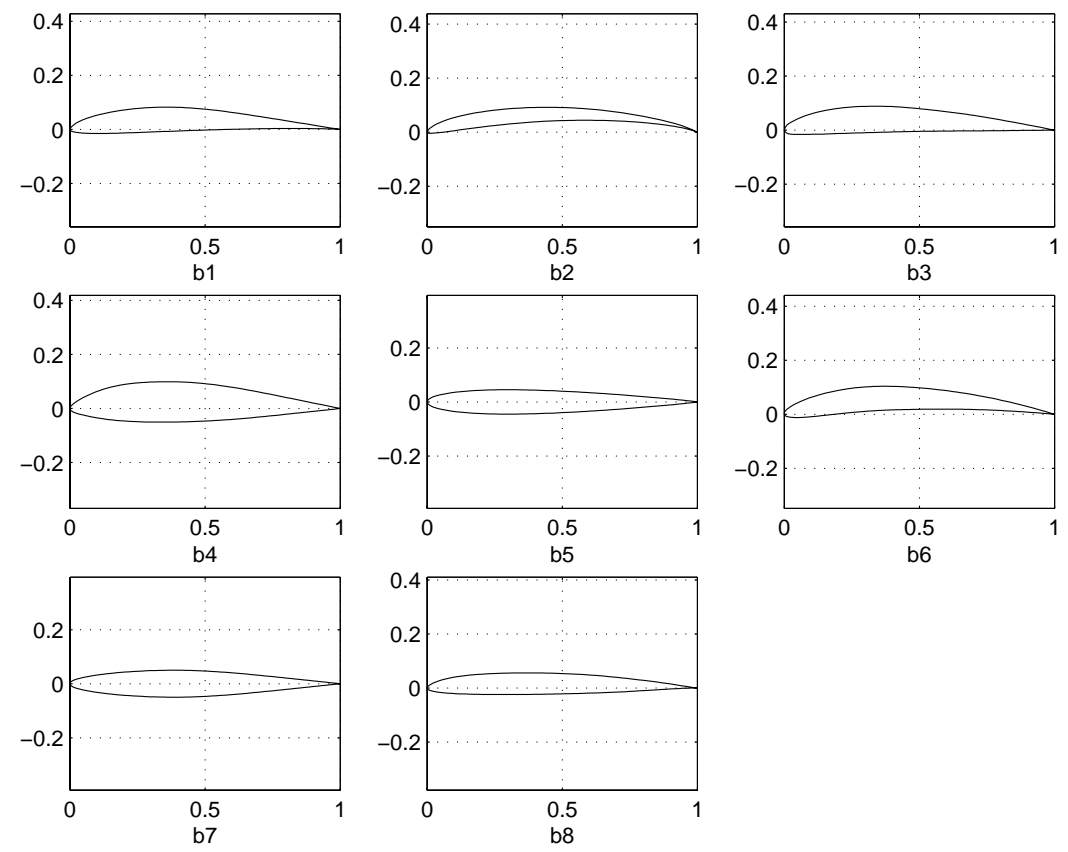

Figure 2. Basis Functions $b_{1}, b_{2}, \ldots, b_{8}$

function $b_{2}$ was clearly dominant. All the others played very little role in the optimization process. Still $b_{6}$ and $b_{8}$ were selected as dominant over the remaining basis functions.

Because basis function $b_{2}$ was dominant over all the other bases in the multiobjective studies, a new set of airfoil basis was required. Before choosing the new basis functions, the next step of this research was to compare conventional UAV Pareto curve to that of the buckle wing.

\section{B. Buckle-Wing Study}

The buckle-wing problem was solved and its Pareto curve established. The three basis functions for this buckle-wing problem are $b_{2}, b_{6}$, and $b_{8}$. The starting point $x_{0}=\left[\begin{array}{llll}0.5 & 0.5 & 0.5 & 1.0\end{array}\right]$. The utopian point $f^{u}=$ [5.0 300]. The convergence tolerances $=1 \mathrm{e}-7$.

Figure 3 represents the buckle-wing's optimized airfoils at the two end points of the Pareto curve. The left side of the figure shows these airfoils in the fused configuration with their $c l / c d_{f u s e d}$ values. The right side shows these airfoils in the split configuration with their $c l_{\text {split }}$ values.

Figure 4 shows the conventional and buckle-wing Pareto curves for comparison. The conventional problem generated Pareto points between $(142.8,1.79)$ and $(155.5,1.65)$. The buckle-wing problem generated Pareto points between $(142.8,2.55)$ and $(156.4,2.31)$. A linear interpolating curve was used to generate the two Pareto curves. The Pareto shift is evident from the two curves. $c l$ values varied from 1.65 to 2.31 in the conventional problem and they varied from 2.31 to 2.55 in the buckle-wing problem. Note that the buckle wing is able to obtain the same $c l / c d$ values. The average $c l$ difference was calculated where the two Pareto domains overlap. The average $c l$ improvement between the two curves is 0.74 or $42.5 \%$.

The optimization problems were not very interesting because most of the design variables (i.e., basis functions) were zeros. Two of the three airfoils comprising the problems' basis functions were disappointing. With the exception of $b_{2}$ the Eppler 61 airfoil, the basis functions were poor selections for a high $\mathrm{cl}$. Therefore, research continued with an extended goal to find a more interesting design problem (i.e. nonzero design variables in the solution) and higher $c l$ results. Further research continued to bolster the project's success. 

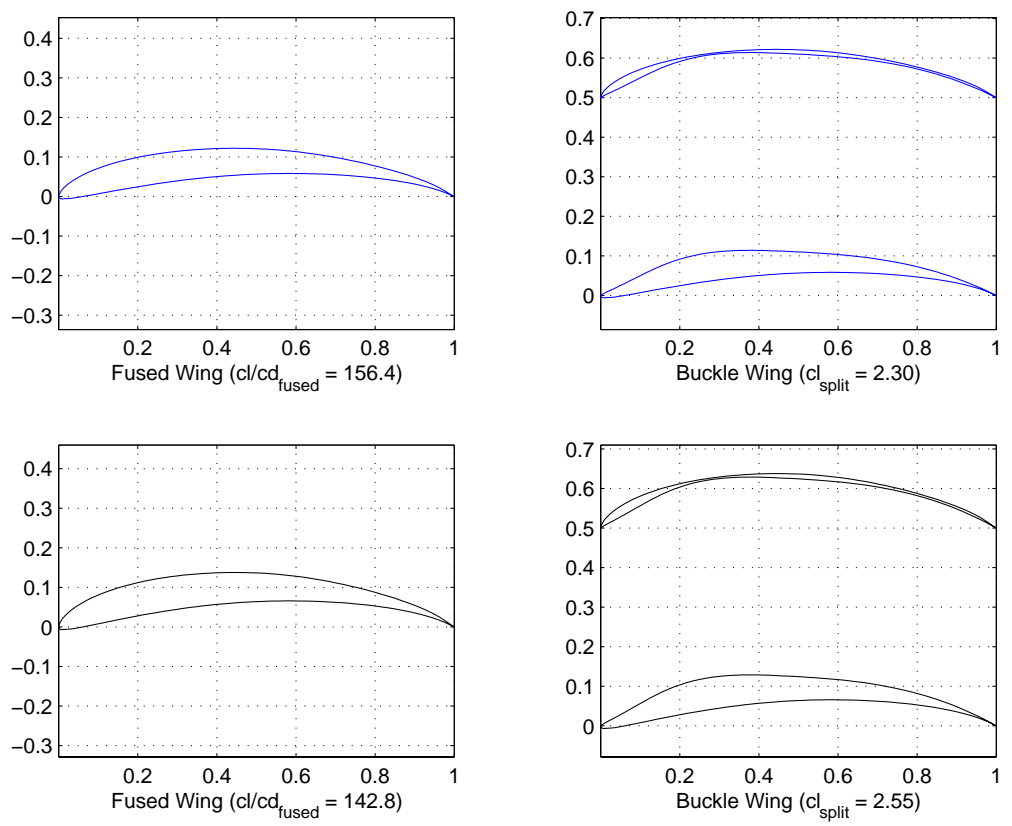

Figure 3. Optimized Buckle-Wing Shapes in Both Configurations

\section{Alternate Airfoil Basis Study for Conventional UAV Design}

The new goal was to find a better buckle-wing UAV design based on two new criteria. First, the design should produce higher $c l$ and $c l / c d$ results. Second, the optimization problem should involve all three basis functions in the optimization process. Each applicable family of airfoils from the website

http://www.aae.uiuc.edu/m-selig/ads/cood_database.html was opened and analyzed visually. The family or series of airfoils, which passed the first visual check of having a thin asymmetric, cambered shape, was then scrutinized more thoroughly. The next visual checks involved comparing cambers. These visual checks were based on experience and intuition from the previous results. Finally, eight new airfoils were selected for high lift. Figure 5 shows these airfoil shapes and names. These eight comprised the alternate airfoil basis design problem.

Table 2 depicts the criteria used in selecting the most dominant three. Clearly, $x_{3}, x_{7}$, and $x_{8}$ were the most frequently used. The corresponding names of these three dominating airfoils are E-61, GOE804, and fxmod74. In this study one airfoil did not dominate all eight, but three played important roles in generating the Pareto curve. In fact, $x_{1}$ played a minor role as well. These three airfoils provided the basis functions for the buckle-wing problem and Pareto curve comparison.

Table 2. Nonzero Weights for the Conventional Alternate Study - 8 Bases Optimization Trials

\begin{tabular}{|l|c|c|c|c|c|c|c|c|}
\hline Basis Function & $x_{1}$ & $x_{2}$ & $x_{3}$ & $x_{4}$ & $x_{5}$ & $x_{6}$ & $x_{7}$ & $x_{8}$ \\
\hline Pareto Trials (18) & 5 & 0 & 15 & 1 & 1 & 1 & 13 & 16 \\
\hline Total Trials (37) & 11 & 4 & 32 & 6 & 5 & 4 & 26 & 35 \\
\hline
\end{tabular}

These three airfoils were among the best airfoil shapes for lift. The Eppler 61 is the best of the Eppler airfoil shapes for lift. Gottingen (EA 8) 804 proved to be the best airfoil basis function from that Gottingen 


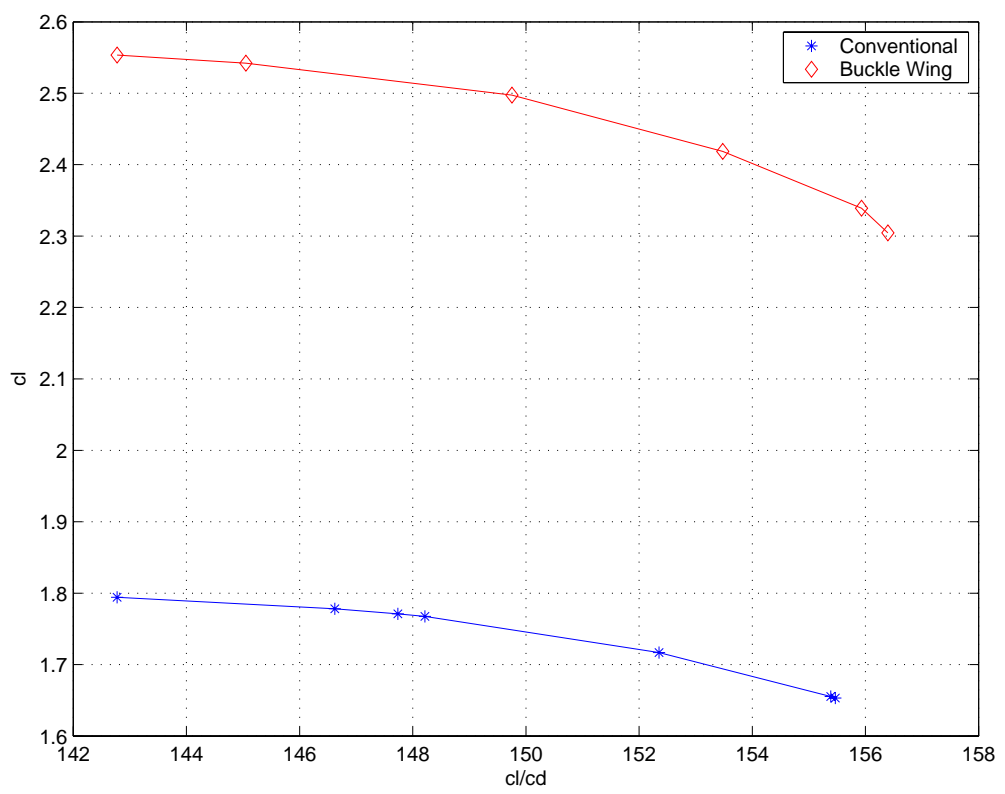

Figure 4. Pareto Curves with Bases $b_{2}, b_{6}, b_{8}$

for lift. Wortmann made modifications to its FX 74-CL5-140 high lift airfoil for even higher lift. Thus, some of the best designs were achieved.

\section{Conventional UAV Study with Three Basis Functions $x_{3}, x_{7}$, and $x_{8}$}

The Pareto curve for the conventional UAV design was generated using the three dominant bases. Tables 3 and 4 display the complete results for this problem. The Pareto curve of the this problem was established for comparison. Figure 6a illustrates the two Pareto curves for conventional design using both eight and three bases. For the most part the two curves are identical; however, the eight-bases case is able to generate better results in two areas of the curve. This was anticipated from the small contributing role that the other five basis functions played in the optimization process and basis function $x_{1}$ in particular.

Using the alternate set of airfoil bases, the Pareto curve is observed to be superior to that of the original problem shown in Figure 6b. The optimizer was able to achieve the same domain for $\mathrm{cl} / \mathrm{cd}$ up to a maximum value at 155.5. Its range for $c l$ well surpasses 1.79. It achieves $c l$ values through 2.15. Furthermore, its $c l$ surpasses every section where the two curves' domain overlap.

\section{E. Buckle-Wing Problem with Alternate Bases}

The buckle-wing problem using the alternate airfoil bases was solved using the knowledge learned from the previous problems. The same starting point, bounds, utopian point, and tolerances were used. The optimizer converged at local minima for nearly half of the trials. Therefore, other starting points were used to find the global minima for these problematic trials. The complete results are found in the Tables 5,6 , and 7 . The Pareto curve was established and compared to the conventional UAV design.

Figure 6c shows the successful Pareto curve shift. The conventional problem generated Pareto points between $(115.9,2.15)$ and $(155.5,1.66)$. The buckle-wing generated points between $(99.9,3.37)$ and $(154.7$, 2.41). Each Pareto curve covered approximately the same $c l / c d$ domain. The curves were constructed using a linear piecewise fit. The average $c l$ improvement between the two curves was 0.803 or $41.7 \%$. 

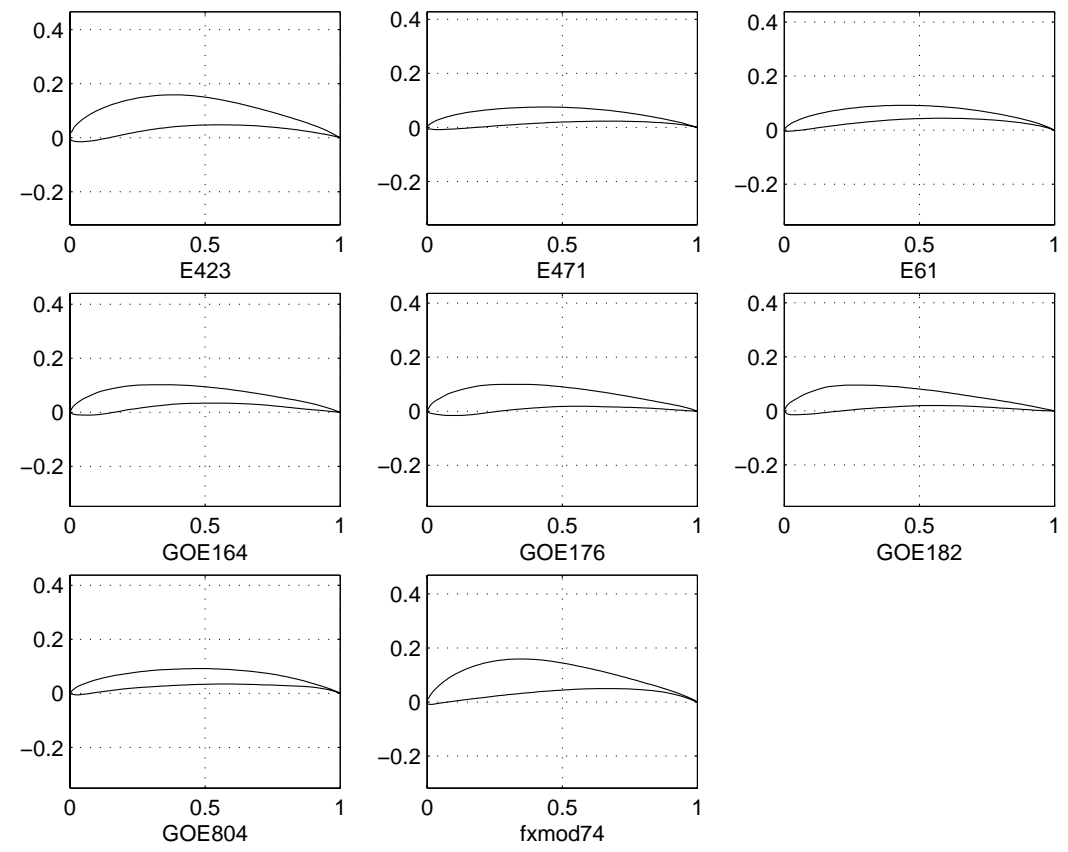

Figure 5. Eight New Basis Functions $x_{1}, x_{2}, \ldots, x_{8}$

Compared to the first buckle-wing trial using the original bases, this buckle-wing Pareto curve was able to generate significantly larger $c l$ values and a larger gamut for the curve. Figure 6d compares the two buckle-wing curves. Using alternate bases the buckle-wing Pareto curve produced higher $c l$ values.

\section{References}

${ }^{1}$ Bowman, J., Sanders, B., Weisshaar, T. Evaluating the Impact of Morphing Technologies on Aircraft Performance, AIAA 2002-1631, 2002.

${ }^{2}$ Gano, S.E., Renaud, J.E., Batill, S.M., Tovar, A., Shape Optimization for Conforming Airfoils, Proceedings of the 44th AIAA/ASME/ASCE/AHS Structures, Structural Dynamics, and Materials Conference, AIAA 2003-1579, Norfolk, VA, April 7-10, 2003.

${ }^{3}$ Kuethe, A.M., Chow, C.Y., Foundations of Aerodynamics Bases of Aerodynamic Design, 5th Edition, John Wiley \& Sons Inc., New York, 1998.

${ }^{4}$ Moran, J., Theoretical and Computational Aerodynamics, John Wiley \& Sons Inc., 1984.

${ }^{5}$ Peters, C., Roth B., Crossley, W.A., Weisshaar, T.A., Use of Design Methods to Generate and Develop Missions for Morphing Aircraft, AIAA 2002-5468, 2002.

${ }^{6}$ Roth, B., Peters, C., Crossley, W.A., Aircraft Sizing with Morphing as an Independent Variable: Motivation, Strategies and Investigations. AIAA 2002-5840, 2002.

${ }^{7}$ Squire, H.B., Young, A.D., The calculation of the Profile Drag of Aerofoils, ARC RM, 1938. 1986.

${ }^{8}$ Steuer, R.E. Multiple Criteria Optimization: Theory, Computation, and Application, John Wiley \& Sons Inc., New York,

${ }^{9}$ Tappeta, R.V., Renaud, J.E., Multiobjective Collaborative Optimization, Journal of Mechanical Design, Vol. 119, No. 3, 1997, pp.403-411.

${ }^{10}$ Vanderplaats, G.N. Numerical Optimization Techniques for Engineering Design, McGraw-Hill, 1984.

${ }^{11}$ Wall R., DARPA Eyes Materials for 'Morphing' Aircraft, Aviation Week and Space Technology, p.36, April 8, 2002.

${ }^{12}$ Wauquiez, C., Shape Optimization of Low Speed Airfoils using MATLAB and Automatic Differentiation, PhD Thesis, Royal Institute of Technology, Department of Numerical Analysis and Computing Science, Stockholm, Sweden, 2000.

${ }^{13}$ Wlezien, R.W., Horner, G.C., McGowan, A.R., Padula, S.L., Scott, M.A., Silcox, R.H., Simpson, J.O., The Aircraft Morphing Program, AIAA 98-1927, April 1998.

10 OF 15 


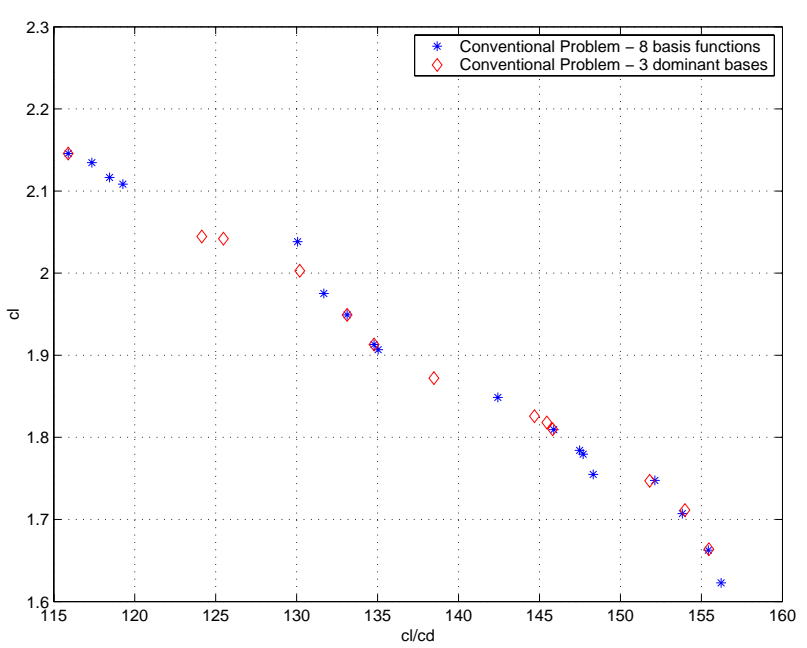

(a) Pareto Curves with High Lift Basis Functions

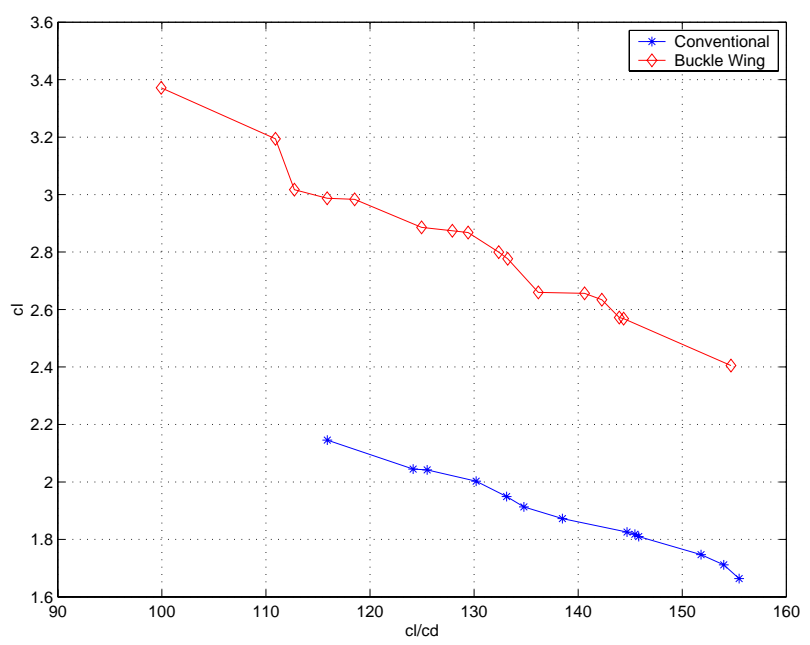

(c) Pareto Curves with bases E61, GOE804, fxmod74

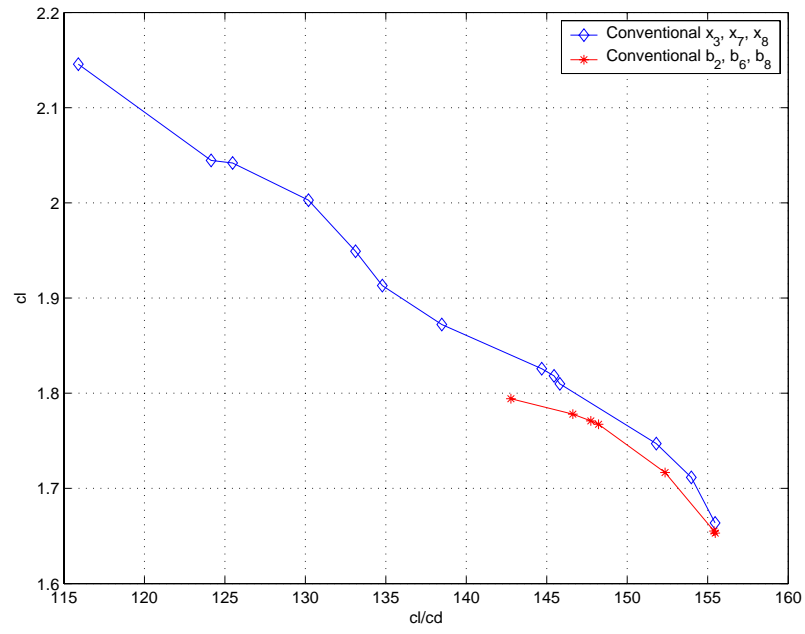

(b) Conventional Pareto Curves with Different Basis Functions

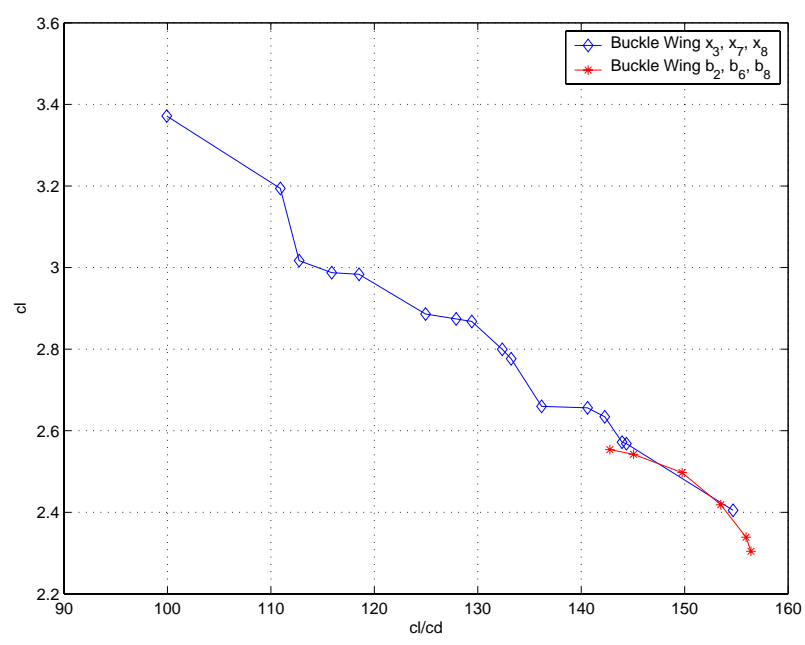

(d) Buckle-Wing Pareto Curves

Figure 6. Pareto Curve Comparisons 
Table 3. Conventional Study - 3 Alternate Bases, Starting Point $x_{0}=\left[\begin{array}{l}0.5 \\ 0.5\end{array}\right.$ 0.5 1.0] Unless Otherwise Specified

\begin{tabular}{|c|c|c|c|c|c|c|c|c|}
\hline $\bar{f}$ & $\mathrm{cl}$ & $c l / c d$ & $x_{1}$ & $x_{2}$ & $x_{3}$ & $x_{4}$ & Pareto & Cons \\
\hline$\left[\begin{array}{ll}1.4 & 150\end{array}\right]$ & 1.4713 & 149.3994 & 0.5214 & 0.5303 & 0.1080 & 1.0120 & no & 15 \\
\hline$\left[\begin{array}{ll}1.4 & 160\end{array}\right]$ & 1.4044 & 155.2235 & 1.1086 & 0.0190 & 0.0000 & 1.1194 & no & 3,15 \\
\hline$\left[\begin{array}{ll}1.4 & 170\end{array}\right]$ & 1.3966 & 155.1372 & 1.0851 & 0.0353 & 0.0000 & 1.4954 & no & 3,15 \\
\hline$\left[\begin{array}{ll}1.5 & 140\end{array}\right]$ & 1.7356 & 149.2438 & 0.9124 & 0.4448 & 0.0676 & 0.8459 & no & 15 \\
\hline$\left[\begin{array}{ll}1.5 & 150\end{array}\right]$ & 1.4555 & 149.3478 & 0.4721 & 0.4721 & 0.1070 & 1.0296 & no & 14 \\
\hline \multicolumn{9}{|c|}{$x_{0}=\left[\begin{array}{llll}.91 & .445 & .0676 & 1\end{array}\right]$} \\
\hline$\left[\begin{array}{ll}1.5 & 150\end{array}\right]$ & 1.6637 & 155.4560 & 1.0673 & 0.2864 & 0.0179 & 0.8909 & yes & 15 \\
\hline \multicolumn{9}{|c|}{$f^{u}=\left[\begin{array}{lll}4 & 300\end{array}\right]$ and $x_{0}=\left[\begin{array}{llll}.91 & .445 & .0676 & 1\end{array}\right]$} \\
\hline$\left[\begin{array}{ll}1.5 & 140\end{array}\right]$ & 1.6746 & 150.9383 & 0.8859 & 0.4216 & 0.0610 & 0.9316 & no & 15 \\
\hline$\left[\begin{array}{ll}1.5 & 150\end{array}\right]$ & 1.6803 & 150.7933 & 0.8743 & 0.4250 & 0.0711 & 0.9947 & no & 15 \\
\hline \multicolumn{9}{|c|}{$f^{u}=\left[\begin{array}{ll}5 & 400\end{array}\right]$ and $x_{0}=\left[\begin{array}{llll}.91 & .445 & .0676 & 1\end{array}\right]$} \\
\hline$\left[\begin{array}{ll}1.5 & 140\end{array}\right]$ & 1.7047 & 150.0777 & 0.8837 & 0.4417 & 0.0693 & 0.9709 & no & none \\
\hline \multicolumn{9}{|c|}{ 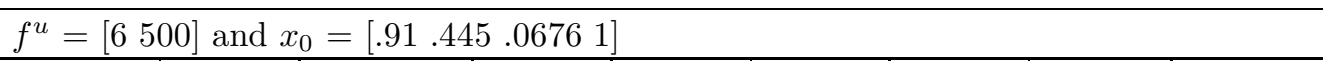 } \\
\hline$\left[\begin{array}{ll}1.5 & 140\end{array}\right]$ & 1.5950 & 147.5961 & 0.7501 & 0.4349 & 0.0954 & 0.9789 & no & 14,15 \\
\hline$\left[\begin{array}{ll}1.6 & 140\end{array}\right]$ & 1.6768 & 143.2924 & 1.1583 & 0.0000 & 0.1689 & 0.9451 & no & $2,14,15$ \\
\hline$\left[\begin{array}{ll}1.6 & 150\end{array}\right]$ & 1.4982 & 149.2095 & 0.4596 & 0.6356 & 0.0957 & 1.0727 & no & none \\
\hline$\left[\begin{array}{ll}1.6 & 160\end{array}\right]$ & 1.4232 & 154.9497 & 1.1452 & 0.0000 & 0.0000 & 1.1263 & yes & $2,3,14,15$ \\
\hline$\left[\begin{array}{ll}1.6 & 170\end{array}\right]$ & 1.3847 & 154.9758 & 1.0495 & 0.0600 & 0.0000 & 1.5008 & no & none \\
\hline \multicolumn{9}{|c|}{$x_{0}=\left[\begin{array}{lllll}1 & 0.4 & 0.1 & 1\end{array}\right]$} \\
\hline$\left[\begin{array}{ll}1.6 & 150\end{array}\right]$ & 1.7115 & 153.9819 & 1.0907 & 0.2708 & 0.0046 & 0.9204 & yes & 14,15 \\
\hline $\left.\begin{array}{lll}1.7 & 140\end{array}\right]$ & 1.8182 & 145.4517 & 1.3446 & 0.0000 & 0.1315 & 0.9091 & yes & 2,15 \\
\hline$\left[\begin{array}{ll}1.7 & 150\end{array}\right]$ & 1.4724 & 141.3505 & 0.4096 & 0.6098 & 0.1335 & 1.1751 & no & 14 \\
\hline$\left[\begin{array}{ll}1.7 & 160\end{array}\right]$ & 1.4161 & 153.4753 & 0.7437 & 0.3168 & 0.0608 & 1.2184 & no & none \\
\hline \multicolumn{9}{|c|}{$x_{0}=\left[\begin{array}{llll}1.1334 & .2730 & .0379 & 1\end{array}\right]$} \\
\hline$\left[\begin{array}{ll}1.7 & 140\end{array}\right]$ & 1.8090 & 145.0327 & 1.1885 & 0.2583 & 0.0520 & 0.9161 & no & $14,15,16$ \\
\hline$\left[\begin{array}{ll}1.7 & 150\end{array}\right]$ & 1.7470 & 151.8031 & 1.1334 & 0.2730 & 0.0379 & 1.0000 & yes & none \\
\hline
\end{tabular}


Table 4. Conventional Study - 3 Alternate Bases, continued

\begin{tabular}{|c|c|c|c|c|c|c|c|c|}
\hline $\bar{f}$ & $\mathrm{cl}$ & $c l / c d$ & $x_{1}$ & $x_{2}$ & $x_{3}$ & $x_{4}$ & Pareto & Cons \\
\hline \multicolumn{9}{|c|}{$x_{0}=\left[\begin{array}{lllll}1.4775 & 0 & 0 & 1\end{array}\right]$} \\
\hline$\left[\begin{array}{ll}1.7 & 140\end{array}\right]$ & 1.8182 & 145.4517 & 1.3446 & 0.0000 & 0.1315 & 0.9091 & (used) & 2,15 \\
\hline$\left[\begin{array}{ll}1.7 & 150\end{array}\right]$ & 1.7440 & 151.6906 & 1.1809 & 0.2585 & 0.0113 & 0.9662 & no & 14,15 \\
\hline$\left[\begin{array}{lllll}1 & 0 & 130\end{array}\right]$ & 10401 & יר21 122 & ( & 00240 & 05244 & 00554 & rec & 11415 \\
\hline$\left[\begin{array}{ll}1.9 & 140\end{array}\right]$ & 1.8462 & 137.0174 & 0.2806 & 0.7243 & 0.4082 & 1.0497 & no & 15 \\
\hline$\left[\begin{array}{ll}1.9 & 150\end{array}\right]$ & 1.8098 & 145.8147 & 1.3364 & 0.0000 & 0.1316 & 1.0837 & yes & none \\
\hline \multicolumn{9}{|c|}{$x_{0}=\left[\begin{array}{llll}1.3364 & 0 & .1316 & 1\end{array}\right]$} \\
\hline$\left[\begin{array}{ll}1.9 & 150\end{array}\right]$ & 1.8257 & 144.6893 & 1.3483 & 0.0000 & 0.1341 & 1.0354 & yes & $2,14,15$ \\
\hline$\left[\begin{array}{ll}1.9 & 140\end{array}\right]$ & 1.8722 & 138.4813 & 1.2897 & 0.0000 & 0.2103 & 1.0253 & yes & $2,14,15,16$ \\
\hline$\left[\begin{array}{ll}2.0 & 120\end{array}\right]$ & 2.0446 & 124.1472 & 0.2480 & 0.5618 & 0.6902 & 0.9554 & yes & 14,16 \\
\hline$\left[\begin{array}{ll}2.0 & 130\end{array}\right]$ & 2.0028 & 130.1928 & 0.0004 & 0.9083 & 0.5913 & 0.9972 & yes & $14,15,16$ \\
\hline$\left[\begin{array}{ll}2.0 & 140\end{array}\right]$ & 1.9130 & 134.7814 & 0.0000 & 0.9346 & 0.5091 & 1.0870 & yes & 14,15 \\
\hline$\left[\begin{array}{ll}2.1 & 110\end{array}\right]$ & 2.1457 & 115.8915 & 0.5613 & 0.0000 & 0.9387 & 0.9492 & yes & $2,10,14,16$ \\
\hline$\left[\begin{array}{ll}2.1 & 120\end{array}\right]$ & 2.0995 & 115.0208 & 0.1737 & 0.4767 & 0.8451 & 1.0006 & no & NR \\
\hline$\left[\begin{array}{ll}2.1 & 130\end{array}\right]$ & 2.0419 & 125.4845 & 0.2267 & 0.5891 & 0.6842 & 1.0645 & yes & NR \\
\hline$\left[\begin{array}{ll}2.3 & 110\end{array}\right]$ & 2.1457 & 115.8915 & 0.5613 & 0.0000 & 0.9387 & 1.2204 & $\mathrm{x}$ & $2,10,14,16$ \\
\hline$\left[\begin{array}{ll}2.3 & 120\end{array}\right]$ & 2.1457 & 115.8915 & 0.5613 & 0.0000 & 0.9387 & 1.2204 & $\mathrm{x}$ & $2,10,14,16$ \\
\hline$\left[\begin{array}{ll}2.3 & 130\end{array}\right]$ & 2.1457 & 115.8915 & 0.5613 & 0.0000 & 0.9387 & 1.2204 & $\mathrm{x}$ & $2,10,14,16$ \\
\hline $\begin{array}{l}F=x_{4}- \\
f^{u}=[3.0 \\
\text { Converge } \\
\text { Constrain } \\
\text { Constrain } \\
\text { Constrain } \\
\text { Constrain } \\
\text { Constrain } \\
\text { Constrain } \\
\text { Constrain } \\
\text { Constrain } \\
\text { Constrain } \\
\text { Constrain }\end{array}$ & $\begin{array}{l}\alpha\left(w_{1} f_{1}+\right. \\
\text { 200] Unl } \\
\text { ce Toler } \\
\text { s 1-4: L } \\
\text { s } 5-8: \mathrm{U} \\
9: 0 \geq \\
\text { 10: } 0.2 \\
11: 0.2 \\
12: 0.3 \\
13:-0 . \\
14: x_{4} \\
15: x_{4} \\
16: 1.5\end{array}$ & $\begin{array}{l}\left.w_{2} f_{2}\right), \\
\text { ss Otherw } \\
\text { nces }=1 \mathrm{e}- \\
\text { wer Bounc } \\
\text { per Boun } \\
m, \\
\geq \max \left(y_{n e}\right. \\
\geq-\min \left(y_{1}\right. \\
\geq \max \left(y_{n e}\right. \\
1 \geq-\max \\
\geq w_{1}\left(f_{1}^{u}-\right. \\
\geq w_{2}\left(f_{2}^{u}-\right. \\
\geq x_{1}+x_{2}\end{array}$ & $\begin{array}{l}w_{i}=1 / \\
\text { e Specif } \\
=[\overline{0}] \\
=[1.5 \\
), \text { where } \\
w), \\
\text { ) }- \text { min } \\
\text { (new })+1 \\
\text { 1) } \\
\text { 2) } \\
x_{3}\end{array}$ & $\begin{array}{l}\left.f_{i}^{u}-\bar{f}_{i}\right) \\
\text { d } \\
\text { tive Col } \\
.51 .53 . \\
y_{\text {new }} \text { is } \\
\left.y_{\text {new }}\right), \\
\operatorname{in}\left(y_{\text {new }}\right.\end{array}$ & straints & $y$ coor & nate, & \\
\hline
\end{tabular}

13 OF 15

American Institute of Aeronautics and Astronautics 


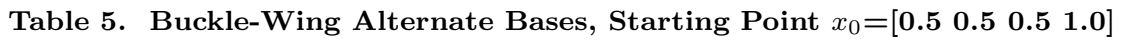

\begin{tabular}{|c|c|c|c|c|c|c|c|c|}
\hline $\bar{f}$ & $c l$ & $\mathrm{cl} / \mathrm{cd}$ & $x_{1}$ & $x_{2}$ & $x_{3}$ & $x_{4}$ & Pareto & Cons \\
\hline $\left.\begin{array}{lll}2.0 & 140\end{array}\right]$ & 2.1687 & 149.2055 & 0.5803 & 0.5227 & 0.1024 & 0.9438 & no & NR \\
\hline$\left[\begin{array}{ll}2.0 & 150\end{array}\right]$ & 2.0913 & 153.4535 & 0.8615 & 0.3186 & 0.0054 & 0.9770 & no & NR \\
\hline$\left[\begin{array}{ll}2.0 & 160\end{array}\right]$ & 1.9530 & 154.8810 & 0.9137 & 0.1788 & 0.0000 & 1.0366 & yes & NR \\
\hline$\left[\begin{array}{ll}2.0 & 170\end{array}\right]$ & 1.9418 & 154.9397 & 0.8572 & 0.2285 & 0.0000 & 1.1185 & yes & 3,11 \\
\hline$\left[\begin{array}{ll}2.1 & 130\end{array}\right]$ & 2.3440 & 145.4206 & 0.6564 & 0.5968 & 0.0804 & 0.9159 & no & NR \\
\hline$\left[\begin{array}{lll}2.1 & 140\end{array}\right]$ & 2.2026 & 148.9075 & 0.5660 & 0.5689 & 0.0963 & 0.9646 & no & NR \\
\hline$\left[\begin{array}{ll}2.1 & 150\end{array}\right]$ & 2.1591 & 153.0544 & 0.9026 & 0.2789 & 0.0378 & 0.9796 & yes & NR \\
\hline$\left[\begin{array}{lll}2.2 & 150\end{array}\right]$ & 2.1837 & 149.0955 & 0.6111 & 0.5021 & 0.1022 & 1.0060 & no & NR \\
\hline$\left[\begin{array}{lll}2.2 & 160\end{array}\right]$ & 2.0894 & 149.3864 & 0.5841 & 0.4561 & 0.1086 & 1.0758 & no & NR \\
\hline$\left[\begin{array}{lll}2.2 & 170\end{array}\right]$ & 1.9419 & 154.9395 & 0.8573 & 0.2283 & 0.0000 & 1.1159 & yes & 11 \\
\hline$\left[\begin{array}{lll}2.5 & 140\end{array}\right]$ & 2.4421 & 136.2838 & 0.3994 & 0.5490 & 0.3518 & 1.0232 & no & NR \\
\hline$\left[\begin{array}{ll}2.5 & 150\end{array}\right]$ & 2.4798 & 148.7860 & 0.8483 & 0.5087 & 0.0718 & 1.0081 & yes & NR \\
\hline$\left[\begin{array}{ll}2.5 & 160\end{array}\right]$ & 2.2790 & 147.6265 & 0.5855 & 0.6172 & 0.0852 & & no & NR \\
\hline$\left[\begin{array}{ll}2.5 & 170\end{array}\right]$ & 2.1109 & 148.6320 & 0.3087 & 0.7843 & 0.0836 & 1.1644 & no & 11 \\
\hline$\left[\begin{array}{ll}2.6 & 130\end{array}\right]$ & 2.6790 & 135.5930 & 0.4102 & 0.6195 & 0.4118 & 0.9671 & no & $\mathrm{NR}$ \\
\hline$\left[\begin{array}{lll}2.6 & 140\end{array}\right]$ & 2.6341 & 142.2728 & 1.3279 & 0.0000 & 0.1663 & 0.9858 & yes & $2,10,11$ \\
\hline$\left[\begin{array}{ll}2.6 & 150\end{array}\right]$ & 2.3936 & 137.1001 & 0.3947 & 0.5819 & 0.3074 & 1.0860 & no & 10,11 \\
\hline$\left[\begin{array}{lll}2.7 & 130\end{array}\right]$ & 2.7423 & 133.3630 & 0.0838 & 0.9544 & 0.4407 & 0.9816 & yes & NR \\
\hline$\left[\begin{array}{lll}2.7 & 140\end{array}\right]$ & 2.6510 & 136.5926 & 0.2280 & 0.7859 & 0.4109 & 1.0213 & no & NR \\
\hline$\left[\begin{array}{lll}2.7 & 150\end{array}\right]$ & 2.5719 & 143.9487 & 0.8267 & 0.6176 & 0.0556 & 1.0557 & yes & NR \\
\hline$\left[\begin{array}{ll}2.7 & 160\end{array}\right]$ & 2.3395 & 138.0592 & 0.4058 & 0.5463 & 0.2981 & 1.1567 & no & 10,11 \\
\hline$\left[\begin{array}{lll}2.9 & 120\end{array}\right]$ & 2.8932 & 124.0234 & 0.4272 & 0.4280 & 0.6449 & 1.0034 & no & 11 \\
\hline$\left[\begin{array}{lll}2.9 & 130\end{array}\right]$ & 2.8742 & 127.9077 & 0.1349 & 0.6889 & 0.6604 & 1.0123 & yes & 11 \\
\hline$\left[\begin{array}{lll}2.9 & 140\end{array}\right]$ & 2.7997 & 132.3587 & 0.0000 & 0.9256 & 0.5512 & 1.0478 & yes & $1,10,11$ \\
\hline$\left[\begin{array}{ll}3.0 & 120\end{array}\right]$ & 2.9836 & 118.5199 & 0.7035 & 0.0000 & 0.7965 & 1.0082 & yes & NR \\
\hline$\left[\begin{array}{ll}3.0 & 130\end{array}\right]$ & 2.8861 & 124.9580 & 0.4291 & 0.4387 & 0.6323 & 1.0571 & yes & NR \\
\hline$\left[\begin{array}{ll}3.0 & 140\end{array}\right]$ & 2.8678 & 129.4216 & 0.0296 & 0.8576 & 0.6128 & 1.0661 & yes & NR \\
\hline$\left[\begin{array}{ll}3.0 & 150\end{array}\right]$ & 2.7764 & 133.2331 & 0.0000 & 0.9304 & 0.5360 & 1.1118 & yes & NR \\
\hline$\left[\begin{array}{ll}3.0 & 160\end{array}\right]$ & 2.6596 & 136.1690 & 0.4624 & 0.6031 & 0.3758 & 1.1702 & yes & 10,11 \\
\hline$\left[\begin{array}{ll}3.2 & 110\end{array}\right]$ & 3.1940 & 110.9140 & 0.3206 & 0.0000 & 1.1794 & 1.0034 & yes & NR \\
\hline$\left[\begin{array}{ll}3.2 & 120\end{array}\right]$ & 3.0731 & 107.3064 & 0.0000 & 0.0000 & 1.3497 & 1.0705 & no & NR \\
\hline$\left[\begin{array}{ll}3.2 & 130\end{array}\right]$ & 3.0171 & 112.7285 & 0.2966 & 0.0000 & 1.1077 & 1.1016 & yes & 11 \\
\hline$\left[\begin{array}{lll}3.2 & 140\end{array}\right]$ & 3.1940 & 110.9140 & 0.3206 & 0.0000 & 1.1794 & 1.0034 & no & NR \\
\hline$\left[\begin{array}{lll}3.2 & 150\end{array}\right]$ & 3.0731 & 107.3064 & 0.0000 & 0.0000 & 1.3497 & 1.0705 & no & NR \\
\hline$\left[\begin{array}{ll}3.2 & 160\end{array}\right]$ & 3.0171 & 112.7285 & 0.2966 & 0.0000 & 1.1077 & 1.1016 & (used) & NR \\
\hline$\left[\begin{array}{ll}3.2 & 170\end{array}\right]$ & 2.9874 & 115.8806 & 0.2397 & 0.4421 & 0.8182 & 1.1507 & yes & 11 \\
\hline
\end{tabular}

continued next page

14 OF 15

American Institute of Aeronautics and Astronautics 
Table 6. Buckle-Wing Alternate Bases, continued

\begin{tabular}{|c|c|c|c|c|c|c|c|c|}
\hline $\bar{f}$ & $c l$ & $\mathrm{cl} / \mathrm{cd}$ & $x_{1}$ & $x_{2}$ & $x_{3}$ & $x_{4}$ & Pareto & Cons \\
\hline$\left[\begin{array}{ll}3.4 & 100\end{array}\right]$ & 3.3714 & 99.9400 & 0.0000 & 0.0000 & 1.5000 & 1.0179 & yes & $2,7,10,11$ \\
\hline$\left[\begin{array}{lll}3.4 & 110\end{array}\right]$ & 3.3129 & 99.6543 & 0.0000 & 0.0000 & 1.4704 & 1.0545 & no & $\mathrm{NR}$ \\
\hline$\left[\begin{array}{lll}3.4 & 120\end{array}\right]$ & 3.2531 & 103.4741 & 0.0020 & 0.0000 & 1.4387 & 1.0918 & no & NR \\
\hline$\left[\begin{array}{ll}3.6 & 90\end{array}\right]$ & 3.3714 & 99.9400 & 0.0000 & 0.0000 & 1.5000 & 1.1633 & (used) & $2,7,10,11$ \\
\hline$\left[\begin{array}{ll}3.6 & 100\end{array}\right]$ & 3.3714 & 99.9400 & 0.0000 & 0.0000 & 1.5000 & 1.1633 & (used) & $2,7,10,11$ \\
\hline$\left[\begin{array}{lll}3.6 & 110\end{array}\right]$ & 3.3714 & 99.9400 & 0.0000 & 0.0000 & 1.5000 & 1.1633 & (used) & $2,7,10,11$ \\
\hline$\left[\begin{array}{ll}3.6 & 120\end{array}\right]$ & 3.3714 & 99.9400 & 0.0000 & 0.0000 & 1.5000 & 1.1633 & (used) & $2,7,10,11$ \\
\hline$\left[\begin{array}{ll}3.6 & 130\end{array}\right]$ & 3.3300 & 97.0000 & NR & NR & NR & NR & no & NR \\
\hline
\end{tabular}

continued below

Table 7. Buckle-Wing Alternate Bases, Starting Point $x_{0}=\left[\begin{array}{llll}1.0 & 0.3 & 0.1 & 1.0\end{array}\right]$

\begin{tabular}{|c|c|c|c|c|c|c|c|c|}
\hline $\bar{f}$ & $\mathrm{cl}$ & $\mathrm{cl} / \mathrm{cd}$ & $x_{1}$ & $x_{2}$ & $x_{3}$ & $x_{4}$ & Pareto & Cons \\
\hline$\left[\begin{array}{ll}2.0 & 140\end{array}\right]$ & 2.2236 & 151.9241 & 0.9271 & 0.3062 & 0.0322 & 0.9255 & no & 10,11 \\
\hline$\left[\begin{array}{ll}2.0 & 150\end{array}\right]$ & 2.0873 & 154.3659 & 1.1809 & 0.0000 & 0.0000 & 0.9709 & no & $2,3,10,11$ \\
\hline$\left[\begin{array}{ll}2.0 & 160\end{array}\right]$ & 1.9941 & 155.1090 & 1.0784 & 0.0399 & 0.0000 & 1.0349 & no & 3,11 \\
\hline$\left[\begin{array}{ll}2.2 & 150\end{array}\right]$ & 2.2258 & 151.3820 & 1.2672 & 0.0077 & 0.0000 & 0.9908 & yes & $3,10,11$ \\
\hline$\left[\begin{array}{ll}2.5 & 130\end{array}\right]$ & 2.6561 & 140.6166 & 1.3081 & 0.0000 & 0.1919 & 0.9375 & yes & NR \\
\hline$\left[\begin{array}{ll}2.5 & 140\end{array}\right]$ & 2.5682 & 144.3618 & 0.8637 & 0.5731 & 0.0588 & 0.9727 & yes & 10,11 \\
\hline$\left[\begin{array}{lll}2.5 & 150\end{array}\right]$ & 2.4795 & 148.7727 & 1.0580 & 0.3287 & 0.0484 & 1.0082 & no & NR \\
\hline$\left[\begin{array}{lll}2.5 & 160\end{array}\right]$ & 2.4049 & 154.6748 & 1.0770 & 0.2905 & 0.0245 & 1.0380 & yes & 10 \\
\hline $\begin{array}{l}\text { Converge } \\
f^{u}=[5.0 \\
\text { Constrain } \\
\text { Constrain } \\
\text { Constrain } \\
\text { Constrain } \\
\text { Constrain } \\
\text { Constrain }\end{array}$ & $\begin{array}{l}\text { ce Toler } \\
\text { 300] } \\
\text { ts } 1-4: \mathrm{L} \\
\text { ts } 5-8: \mathrm{U} \\
9: 0.9 \\
10: x_{4} \\
11: x_{4} \\
12: 1.5 \\
\end{array}$ & $\begin{array}{l}\text { ances }=1 \mathrm{e}- \\
w_{i}=1 /(f \\
\text { wer Boun } \\
\text { pper Boun } \\
\geq c_{f u s e d} \\
\geq w_{1}\left(f_{1}^{u}-\right. \\
\geq w_{2}\left(f_{2}^{u}-\right. \\
\geq x_{1}+x_{2}\end{array}$ & $\begin{array}{l}\left.-\bar{f}_{i}\right) \\
=[\overline{0}] \\
=[1.5\end{array}$ & .51 .53$. & & & & \\
\hline
\end{tabular}

15 OF 15

American Institute of Aeronautics and Astronautics 